Tropical Journal of Pharmaceutical Research September 2019; 18 (9): 1895-1901

ISSN: 1596-5996 (print); 1596-9827 (electronic)

(1) Pharmacotherapy Group, Faculty of Pharmacy, University of Benin, Benin City, 300001 Nigeria.

Available online at http://www.tjpr.org

Original Research Article

http://dx.doi.org/10.4314/tjpr.v18i9.16

\title{
Conjugated linoleic acid attenuates neuropathic pain induced by sciatic nerve in mice
}

\author{
Qingbin Shi*, Xiuying Cai, Changchun Li, Zhen Wang, Xingle Lv \\ Department of Encephalopathy, Zhangqiu District of Traditional Chinese Medicine Hospital, Ji'nan, 250200, China \\ *For correspondence: Email: shiqingbinchxw@163.com
}

Sent for review: 12 June 2019

Revised accepted: 30 August 2019

\begin{abstract}
Purpose: Conjugated linoleic acid (CLA) has been suggested to be necessary for human health, but there is limited research regarding its effect on neuropathic pain (NP). Here, we aim to investigate the potential effect of CLA administration on NP development and nerve recovery.

Methods: Forty mice were divided into four equal groups randomly. The mice in control group underwent a sham operation to achieve a unilateral sciatic nerve cut. Other groups were subjected to partial sciatic nerve ligation (PSNL) surgery followed by 4 weeks of CLA treatment. Behavioral tests were performed shortly before mice were sacrificed. Blood, sciatic nerve and spinal cord tissues were collected after sacrifice. Electron microscopy was performed to determine myelin thickness and calculate myelin thickness/axon diameter ratio.

Results: Mice that received daily oral CLA treatment for 4 weeks after PSNL surgery showed less mechanical and thermal allodynia than mice in PSNL surgery alone group. Behavioral tests showed that CLA treatment was associated with marked increases in both nerve conduction velocity (NCV) and force of gastrocnemius contraction. In addition, CLA reduced the levels of interleukin-1 $\beta$ (IL-1 $\beta$ ) and tumor necrosis factor- $\alpha$ (TNF- $\alpha$ ), sciatic myeloperoxidase (MPO) activity, and activating transcription factor-3 (ATF-3) expression. CLA also restored mitochondrial manganese superoxide dismutase (MnSOD) activity which was decreased in the sciatic nerves and spinal cords of the PSNL surgery group. Regeneration of myelins and axons in nerve fibers in CLA group was faster and more complete than that in the vehicle group.

Conclusion: The current study demonstrates that CLA effectively attenuates NP and significantly inhibits neuro-inflammation and oxidative stress. This treatment improves sciatic nerve form and function after injury, suggesting that it can attenuate NP.
\end{abstract}

Keywords: Conjugated linoleic acid, Nerve pain, Oxidative stress, Neuro-inflammation

\begin{abstract}
This is an Open Access article that uses a fund-ing model which does not charge readers or their institutions for access and distributed under the terms of the Creative Commons Attribution License (http://creativecommons.org/licenses/by/4.0) and the Budapest Open Access Initiative (http://www.budapestopenaccessinitiative.org/read), which permit unrestricted use, distribution, and reproduction in any medium, provided the original work is properly credited.

Tropical Journal of Pharmaceutical Research is indexed by Science Citation Index (SciSearch), Scopus, International Pharmaceutical Abstract, Chemical Abstracts, Embase, Index Copernicus, EBSCO, African Index Medicus, JournalSeek, Journal Citation Reports/Science Edition, Directory of Open Access Journals (DOAJ), African Journal Online, Bioline International, Open-J-Gate and Pharmacy Abstracts
\end{abstract}

\section{INTRODUCTION}

Peripheral nerve trauma is one of the major causes which could lead to motor disability. Even with optimal surgical repair, but the clinical outcome of patients is often poor [1]. Damage to a peripheral nerve usually results in neural cell damage, which can cause allodynia and hyperalgesia [2]. Severe neuropathic pain (NP) usually negatively affects quality of life [3]. After nerve injury occurred, axon degeneration and myelin sheath collapse usually occur within 24 
hours [4]. At the same time, a neuroinflammatory process is initiated, including immune cell recruitment into location of the incident and activation of microglia and astrocytes, which are immune-like cells of the central nervous system (CNS) [5]. These changes induce but not limit to demyelination, remyelination, axonal degeneration, axonal regeneration, nerve fiber damage, and endoneural edema [6]. These maladaptive responses trigger hypersensitivity symptoms such as allodynia, hyperalgesia, and ongoing spontaneous pain [7].

In NP related to peripheral nerve injuries, combined use of vitamin B, nucleotides, folic acid, and polyunsaturated fatty acids promoted nerves regeneration and improve NP-related symptoms $[8,9]$. Conjugated linoleic acid (CLA) has been suggested to be necessary for human health [10] , but there is limited research regarding its potential effect on NP.

This study evaluated the effect of oral CLA treatment on NP progression after partial sciatic nerve ligation (PSNL) surgery in mouse models [11].This model is usually used to study nerve injury and regeneration and to observe morphological changes in axons and myelins in the sciatic nerves.

\section{EXPERIMENTAL}

\section{Mice}

Forty adult male C57BL/6 mice which were weighing from 15 gram to 30 gram were purchased from Jiangsu Biocytogen Co., Ltd. (Nantong, China) and prepared for using in this program. All the protocols treating mice were approved by the Ethical Animal Care Committee of the Zhangqiu District of Traditional Chinese Medicine Hospital (approval no. 20180023) previously. All the mice were kept in a controllable condition at a temperature about 22 - 25을 under a 12 hours' light-dark cycle with food and water ad libitum. Mice were then divided into four experimental groups randomly (10 mice per group): PSNL + vehicle group, PSNL + CLA group, PSNL alone group, normal control group. The experiments with mice were in full compliance with the European Communities Council Directive of 24 November, 1986 (86/609/EEC).

\section{Partial sciatic nerve ligation surgery}

We produced neuropathic pain model through induction using PSNL surgery according to the introduced methods which were described previously[12]. Briefly, the mice were anesthetized using xylazine $(10 \mu \mathrm{g} / \mathrm{g})$ and ketamine $(100 \mu \mathrm{g} / \mathrm{g})$ (intraperitoneally). Then they underwent surgery, which made their left sciatic nerves ligated from one-third to one-half with 4 - 0 catgut sutures at the mid-thigh level. The skin incisions were then repaired with a 5 - 0 nylon suture thread. The sham-operated (control) group had their sciatic nerves exposed out but not been ligated and the sham group received not any chemical treatment.

\section{CLA treatment}

Liquid CLA (HK Biotech Co., Ltd., Jinju, Korea) was given daily for 4 weeks starting on the day of PSNL surgery. The vehicle group received saline (1\% carboxymethyl cellulose) instead. All oral administrations were executed by experienced veterinarian using gavage.

\section{Behavioral tests}

The assessments of mechanical allodynia were performed using von Frey filaments from 1 to $2 \mathrm{~g}$. The procedure was as follows. First, we placed mice on a grid platform which was inside of acrylic boxes for no more than 1 hour per day. This was repeated for three days. Then, we tested their basal withdrawal threshold conventionally before carrying out a surgery, and then we assessed allodynia at 7, 14, and 21 days after the operations. We determined the withdrawal threshold as the thinnest filament which could elicit a nociceptive response in mice. All measures' data were presented as a percentage of the basal values, set at $100 \%$. We assessed the thermal hypernociception using the Hargreaves test. Briefly, we first placed mice on a platform for up to $30 \mathrm{~min}$ per day during for total three days. Then we recorded the latency times for paw withdrawal from the moment a beam of radiant heating on the left plantar surface to the moment eliciting a nociceptive withdrawal response. As the same, the basal withdrawal latency times were recorded before carrying out a surgery, and the hyperalgesia was tested at 7,14 , and 21 days after the operations. All the results were shown as delta $(\Delta)$ of the latency time in seconds.

\section{Cytokine measurement}

Levels of cytokines, including interleukin-1 $\beta$ (IL$1 \beta$ ) and tumor necrosis factor- $\alpha$ (TNF- $\alpha$ ), were quantified using the mouse-specific IL-1 $1 \beta$ and TNF- $\alpha$ enzyme-linked immunosorbent assay (ELISA) kits respectively (eBioscience, San Diego, CA), according to the manufacturer's protocols. Sciatic nerves were detached from 
mice at day 7 after operations, and the they were immersed into 1x Cell Lysis Buffer (Cell Signaling Technologies, Denver, MA), and sonicated for 30 minutes. After centrifugation (5000×g for $15 \mathrm{~min})$, the supernatants were collected and then transferred from the original tubes into fresh tubes for further ELISA assays. All absorbance values were normalized with the absorbance of standards based on the standard curve.

\section{Quantitative real-time polymerase chain reaction (PCR)}

Total RNAs were extracted using TRIzol reagent (Life Technologies, Carlsbad, CA) according to manufacturer's instruction, and then RNAs were digested using DNase (Life Technologies). Next, $1 \mu \mathrm{g}$ of total RNAs were reversely transcribed into cDNA using the QuantiTect Reverse Transcription Kit (Qiagen, Hilden, Germany). Real-time PCR was carried out using the machine, Applied Biosystems StepOne RealTime PCR System, with the regent SYBR Green PCR Master Mix (Applied Biosystems, Foster City, CA), according to the manufacturer's instruction. PCR conditions were as follows: 15 $\mathrm{min}$ at $95^{\circ} \mathrm{C}$, followed by 40 cycles of $15 \mathrm{~s}$ at 95 ${ }^{\circ} \mathrm{C}$ and $30 \mathrm{~s}$ at $60{ }^{\circ} \mathrm{C}$. Finally, the relative quantification method was adopted for assessment of target mRNA expression following the manufacturer's instruction. All measurements were carried out in duplicates. Glyceraldehyde 3phosphate dehydrogenase (GAPDH) gene was used to normalize the relative expression of target mRNAs.

\section{Western blotting analysis}

Proteins were extracted from tissues using lysis buffer and concentration was determined. Then, equal amounts of protein were loaded and separated by $10 \%$ SDS polyacrylamide gels, and were then transblotted onto polyvinylidene difluoride membranes (GE Healthcare, Chicago, IL). After that, the membranes were blocked using $5 \%$ skim milk for 1 hour. Finally, the membranes were incubated with the indicated specific primary antibodies overnight at $4^{\circ} \mathrm{C}$. The next day, the membranes were incubated with appropriate horseradish peroxidase (HRP)conjugated secondary antibodies (dilution 1:10,000; Cell Signaling Technology) for about 1 hour. Then, the membranes were developed using the regent of Pierce ECL Western Blotting Substrate Plus (Waltham, MA).

\section{Statistical analysis}

The data were presented as mean \pm standard deviation (SD), and differences among groups were assessed with one-way analyses of variance (ANOVAs) method. For all analyses, $p$ values less than 0.05 were considered to be significantly different. All statistical analyses were carried out using SPSS 20.0 software (IBM Corp., Armonk, NY).

\section{RESULTS}

CLA prevent the development of mechanical and thermal allodynia after PSNL surgery

Evaluation of mechanical allodynia test and thermal hypernociception test were tested at 7 days after PSNL surgery and CLA treatment initiation. The results showed that CLA treatment prevented mice from thermal hypernociception and mechanical allodynia. That is, CLA-treated mice had significantly higher withdrawal thresholds than PSNL surgery only mice. (Figure $1 \mathrm{~A}$ and $\mathrm{B})$.
A

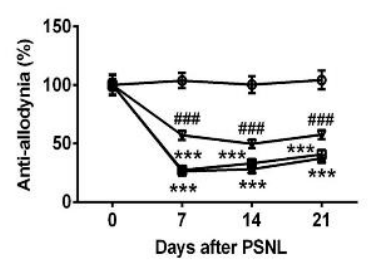

B

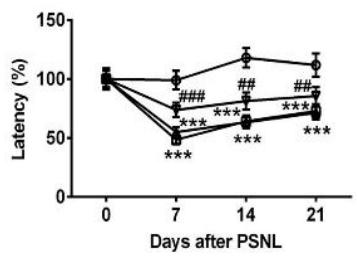

Figure 1: Effect of conjugated linoleic acid (CLA) on partial sciatic nerve ligation (PSNL) surgery-induced mechanical allodynia and thermal hypernociception. Percentages of basal mechanical withdrawal threshold represented as anti-allodynia (A), and latency (B) after daily oral treatment; $n=10 ; \quad$ PSNL + CLA group; $\triangle$ : PSNL + vehicle group; $\square$ : PSNL group; $\circ$ : normal control. Two-way analysis of variance (ANOVA), ${ }^{* * *} p<$ 0.0001 vs. control group, ${ }^{\# \# \# p} 0.0001$ vs. PSNL group

\section{Effect of CLA on nerve conduction velocity (NCV) and force of muscle contraction}

Electrophysiologic recordings revealed that sciatic nerve injury decreased NCV when compared to the control (Figure $2 \mathrm{~A}$ ). A significant impairment in PSNL surgery mice was observed throughout the experiment. CLA treatment significantly increased NCV, when compared to untreated PSNL surgery mice. There was not any significant difference between the CLA and the normal control group. There was also an obvious improvement in the force of muscle contraction in the CLA group when compared to PSNL surgery only group, nearly to the level of the normal control group (Figure $2 \mathrm{~B}$ ).

Regarding changes in body weight, all groups showed similar trends after PSNL surgery. The 
CLA group had a smaller increase when compared to normal control group, similar to the vehicle (PSNL + vehicle) group (Figure $2 \mathrm{C}$ ).
A

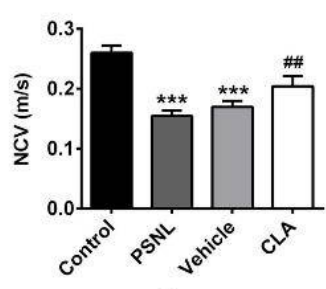

B

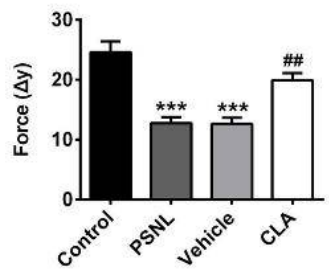

C

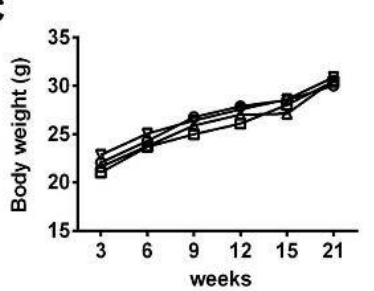

Figure 2: Nerve conduction velocity (NCV) and force of muscle contraction. Comparison of NCV (A) and muscle contraction force (B) between control, partial sciatic nerve ligation (PSNL), vehicle and conjugated linoleic acid (CLA) groups at 4 weeks. (C) Body weights over time. Values expressed as mean \pm standard deviation (SD), $n=8-10 /$ group. $: \mathrm{PSNL}+$ CLA group; $\triangle$ : PSNL + vehicle group; $\square$ : PSNL group; $\circ$ : normal control group. ${ }^{\star \star \star} p<0.0001$ vs. control group, ${ }^{\#} p<0.01$ vs. PSNL group

\section{CLA prevents PSNL surgery-induced increase of pro-inflammatory cytokines}

To examine the mechanism underlying the antihyperalgesic effects of CLA, the levels of proinflammatory cytokines, IL- $1 \beta$ and TNF- $\alpha$, in sciatic nerves, were measured. On day 7 after PSNL surgery, we noted increases in TNF- $\alpha$ in the contra- and ipsilateral sciatic nerves, which were inhibited by CLA treatment (Figure $3 \mathrm{~A}$ ). Similarly, IL-1 $\beta$ levels were increased in the sciatic nerves on both sides in the PSNL surgery group, and this increase was weakened by CLA treatment (Figure $3 \mathrm{~B}$ ). Compared to vehicle treatment, CLA administration did not alter the expression levels of either TNF- $\alpha$ or IL-1 $\beta$ in the sciatic nerve of sham-operated mice, which were referenced as a control group. The effects of CLA on pro-inflammatory cytokine mRNA expression levels were also investigated. As shown in Figures $3 \mathrm{C}$ and $\mathrm{D}$, there were significant increases in the mRNA expression levels of TNF- $\alpha$ and IL-1 $\beta$ in the sciatic nerves of PSNL surgery mice when compared with those of control group.
A

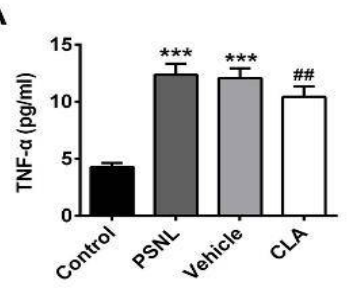

B

C

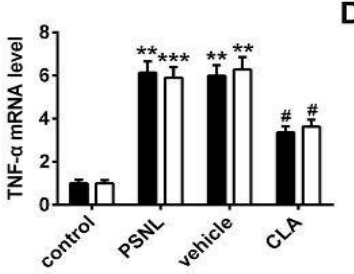

D
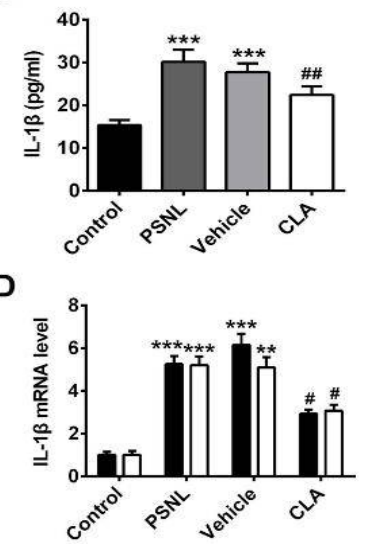

Figure 3: Conjugated linoleic acid (CLA) prevents partial sciatic nerve ligation (PSNL) surgery-induced increases in TNF- $\alpha$ and IL-1 $\beta$ levels. The PSNL surgery-induced increases in (A) TNF- $\alpha$ and (B) IL-1 $\beta$ were mitigated by CLA treatment. PSNL surgery significantly increased expressions of (C) TNF- $\alpha$ and (D) IL-1 $\beta$ mRNA in the sciatic nerve, whereas CLA reduced the levels of these mRNAs $(n=8)$. Data shown as mean \pm standard deviation (SD). $\mathbf{\square}: 7 \mathrm{~d}$, ㅁ: 14d. ${ }^{* \star *} p<0.0001$ vs. control group, ${ }^{\star *} p<0.01$ vs. control group, ${ }^{\#} p<0.01$ vs. PSNL group, ${ }^{p} p<0.05$ vs. PSNL group

CLA attenuate neuro-inflammation by reducing TNF- $\alpha$ levels and sciatic myeloperoxidase (MPO) activity after nerve injury

When injury occurred, neutrophils were among the first kind of immune cells to migrate into and accumulated in the injured areas, initiating the inflammatory responses, and inhibiting hypersensitivity. The activity of myeloperoxidase (MPO) is always used as an indirect marker which could indicate that neutrophils are migrating and accumulated into injured nerves. The results showed that the activity of MPO was highly increased in the sciatic nerves of PSNL surgery mice, but CLA treatment induced a moderate but significant reduction (Figure $4 \mathrm{~A}$ ). CLA treatment also prevented the upregulation of the transcription factor, activation transcription factor 3 (ATF-3), which is a marker of indicating neuronal injury, after PSNL surgery. PSNL surgery-mice receiving the vehicle treatment did not display any significant reduction in ATF-3 expression level when compared to the PSNL surgery only group (Figure $4 \mathrm{~B}$ ).

\section{Positive effect of CLA on superoxide dismutase (SOD) activity in PSNL surgery mice}

To further evaluate the role of CLA in regulating NP-associated oxidative stress, we measured the activity of antioxidant superoxide dismutase 
(SOD), and found that Copper/zinc ( $\mathrm{Cu} / \mathrm{Zn}) \mathrm{SOD}$ activity levels in the spinal cord (SP) and sciatic nerve (SN) were comparable in all four groups (Figure 5A and $\mathrm{B}$ ). In contrast, we found that manganese $(\mathrm{Mn}) \mathrm{SOD}$ activity was significantly decreased on the ipsilateral sides of both the spinal cord and peripheral nerve in PSNL surgery only mice, but normal MnSOD activity was restored by CLA treatment (Figures $5 \mathrm{C}$ and $\mathrm{D}$ ).
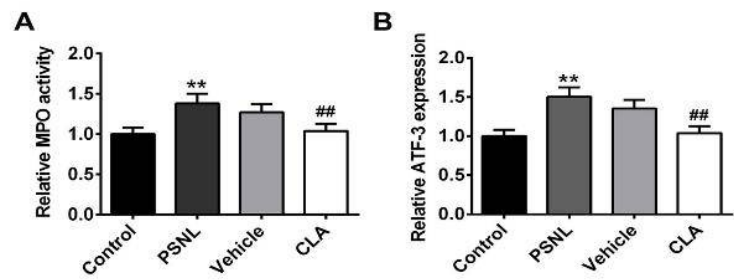

Figure 4: Activity of MPO and the expression level of ATF-3 in partial sciatic nerve ligation (PSNL) surgery. Sciatic nerve MPO activity (A) and ATF-3 expression level (B). $(n=8)$. One-way analysis of variance (ANOVA), ${ }^{\star *} p<0.01$ vs. control group, ${ }^{\# \#} p<0.01$ vs. PSNL group
A

C

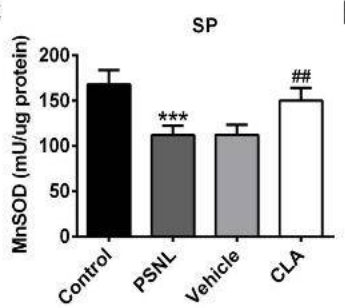

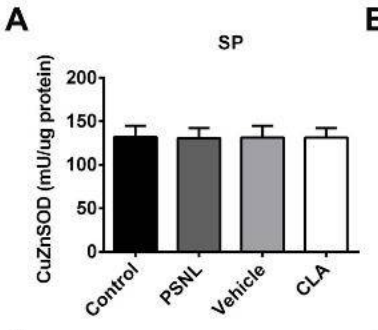

B

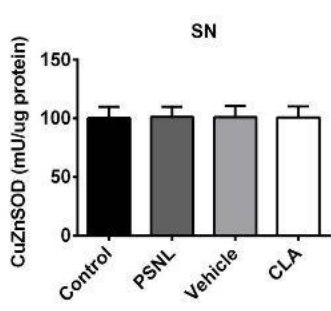

D

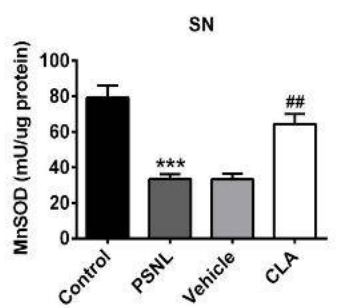

Figure 5: Effect of conjugated linoleic acid (CLA) on superoxide dismutase (SOD) activity in neuropathic pain (NP) mice. Not any changes in Cu/ZnSOD were observed in either the spinal cord (A) or peripheral nerve (B) of partial sciatic nerve ligation (PSNL) surgery mice. CLA treatment had not any marked effect on Cu/ZnSOD activity. Decreased MnSOD activity was shown in both the spinal cord (C) and sciatic nerve (D) of PSNL surgery mice but was restored by CLA treatment $(\mathrm{n}=6) ;{ }^{* \star *} p<0.0001$ vs. control group, ${ }^{\#} p<0.01$ vs PSNL group

\section{Regenerative effect of CLA on sciatic nerve after injury}

The mean axon area of the sciatic nerves in the control (sham only), PSNL (PSNL only), vehicle (PSNL + vehicle), and CLA (PSNL + CLA) groups are shown in Figure 6. Following nerve injury, axon area appeared to be reduced when compared to that of the control group, but it was not statistically significant. In the CLA group, a smaller loss of axon area compared to that of the PSNL surgery group was observed (Figure $6 \mathrm{~A}$ ). There was a larger reduction in axon diameter at day 7 after PSNL surgery when compared to that in the control group, but this loss was smaller in the CLA group (Figure $6 \mathrm{~B}$ ). Figure $6 \mathrm{C}$ shows that the mean myelinated fiber area of the sciatic nerve was markedly reduced in PSNL surgery mice when compared to control mice. A smaller loss of myelinated fiber area was observed in the CLA group when compared to PSNL surgery mice. Similarly, Figure $6 \mathrm{D}$ shows that the mean myelinated area of the sciatic nerve was reduced in PSNL surgery mice when compared to the control group, but this change was attenuated by CLA.
A
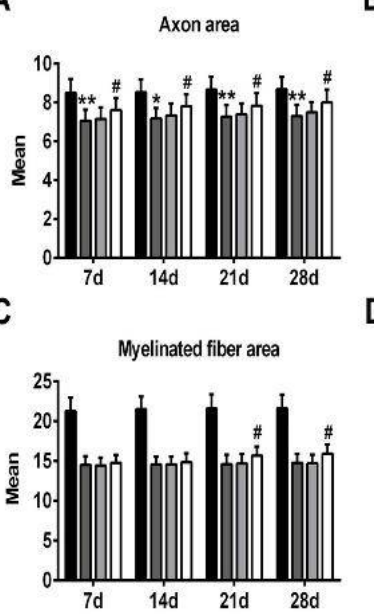
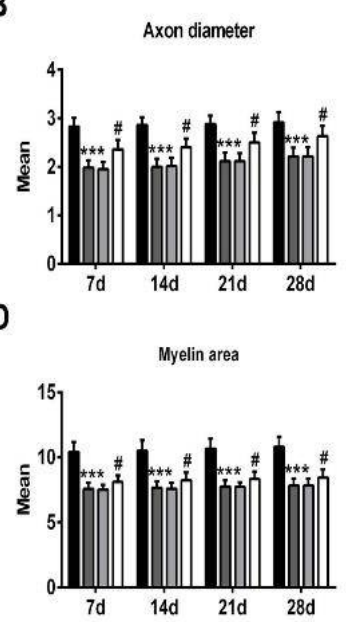

Figure 6: Histological analyses of myelin and axons. (A) Mean axon areas $\left(\mu \mathrm{m}^{2}\right)$ of the sciatic nerves with standard deviations (vertical bars). (B) Mean axon diameters $(\mu \mathrm{m})$ of sciatic nerves with standard deviations (vertical bars). (C) Mean myelinated fiber areas of the sciatic nerves with standard deviations (vertical bars). (D) Mean myelin areas $\left(\mu \mathrm{m}^{2}\right)$ of the sciatic nerves with standard deviations (vertical bars); $\mathrm{n}=6$. One-way analysis of variance (ANOVA) when compared to the conjugated linoleic acid (CLA), control, and partial sciatic nerve ligation (PSNL) groups at each time point. - Control (sham only) group, $\square$ : PSNL (PSNL only) group, $\square$ : Vehicle (PSNL + vehicle) group, $\square$ : CLA (PSNL + CLA) group. ${ }^{* * *} p<0.0001$ vs. control group, ${ }^{* *} p<0.01$ vs. control group, ${ }^{*} p<0.05$ vs. control group, ${ }^{\sharp} p<0.05$ vs. PSNL group

\section{DISCUSSION}

Peripheral nerve injury due to damage or disease affecting somatosensory fibers is one of the main causes of NP[13]. PSNL surgery is a wellestablished method and had been used for mouse model of NP which could mimic impaired 
thermal and mechanical hypersensitivity[14]. Neuropathic pain is the result of sensory nervous system damage that increases pain sensitivity. This study found that 7 days after injury, mice showed consistent thermal hypernociception and mechanical allodynia. However, oral CLA treatment effectively reduced the mechanical withdrawal threshold related to sciatic nerve injury. Conjugated linoleic acid also prevented thermal hypersensitivity, indicating its protective role in NP pathogenesis. In addition to mechanical and thermal nociceptive behavioral analyses, electrophysiological studies showed strong functional recovery, providing evidence that oral CLA supplementation promote the reestablishment of motor and sensory functions after nerve injury.

After nerve damage occurred, immune cells release several mediators, such as chemokines and cytokines that promote neuro-inflammation [15]. The migration of macrophages and neutrophils into injured loci was an essential action that improved peripheral sensitization, and contributed to abnormal neuronal function [15]. Immediately following injury, neutrophils are the first kind of immune cells to migrate to the sites of injury, and amplify inflammatory response signals by recruiting macrophages, leading to NP hypersensitivity. TNF- $\alpha$ is one of the major cytokines contributing to NP due to its effects on driving hypersensitivity development after nerve injuries occurred [16]. The present work showed that CLA decreased TNF- $\alpha$ and IL-1 $\beta$ levels in the sciatic nerve, indirectly evaluated by ATF-3 expression and MPO activity. Neuroinflammation is one of the most important drivers of abnormal neuronal activation after nerve injuries occurred. Activating Transcription Factor 3 is a marker of neuronal injuries which is rapidly induced expression in dorsal root ganglia neurons following injuries occurred [17]. Interestingly, ATF-3 has been reported to express in neurons with successful reinnervation and regeneration, suggesting a connection between ATF-3 and regenerative capacity $[18,19]$.

Among the pro-inflammatory cytokines, TNF- $\alpha$ and IL-1 $\beta$ are predominantly produced by microglia and astrocytes, respectively. Neutralization of TNF- $\alpha$ and IL-1 $\beta$ reduces pain hypersensitivity with NP $[20,21]$. In the current study, PSNL surgery mice showed significant increases in sciatic nerve levels of IL-1 $\beta$ and TNF- $\alpha$, which is consistent with the development of thermal and mechanical allodynia. It is also in accordance with previous studies of elevated IL$1 \beta$ and TNF- $\alpha$ in various NP mouse models $[4,22]$. Oral treatment with CLA suppressed pro- inflammatory cytokine elevations in the injured sciatic nerve. This supports the hypothesis that CLA modulates neuro-inflammation in an NP mouse model.

In NP, nerve injury reduces SOD activity and upregulates the activity and expression of nitric oxide synthase (an enzyme responsible for NO synthesis), leading to increased concentrations of peroxynitrite and subsequent oxidant damage. This work did not find evidence of altered $\mathrm{Cu} / \mathrm{ZnSOD}$ activity in PSNL surgery mice, but decreased MnSOD activity was seen in the spinal cords and sciatic nerves. This was restored by oral administration of CLA significantly.

The histological analyses showed that mean axon area was significantly decreased in the PSNL surgery group. Other morphometric parameters such as the myelinated fiber area and myelinated axon number were decreased in the PSNL surgery group when compared to those of the control group significantly. conjugated linoleic acid treatment prevented these morphometric changes.

\section{CONCLUSION}

Treatment with CLA markedly ameliorates PSNL surgery-induced thermal and mechanical allodynia. Conjugated linoleic acid treatment also significantly attenuates increases in proinflammatory cytokine levels and decreases in SOD activity in PSNL surgery neuropathic mice, indicating that it protects against neuroinflammation and oxidative stress. Daily oral CLA administration enhances the recovery of damaged peripheral nerves, but the mechanism underlying these effects needs to be confirmed in further studies. Thus, the present work suggests that CLA may be an effective and novel treatment for NP caused by nerve injury.

\section{DECLARATIONS}

\section{Conflict of interest}

No conflict of interest is associated with this work.

\section{Contribution of authors}

We declare that this work was done by the authors named in this article and all liabilities pertaining to claims relating to the content of this article will be borne by the authors. QS designed all the experiments and revised the paper. CX, 
WZ and LX performed the experiments. LC and QS wrote the paper.

\section{Open Access}

This is an Open Access article that uses a funding model which does not charge readers or their institutions for access and distributed under the terms of the Creative Commons Attribution License (http://creativecommons.org/licenses/by/ 4.0) and the Budapest Open Access Initiative (http://www.budapestopenaccessinitiative.org/rea d), which permit unrestricted use, distribution, and reproduction in any medium, provided the original work is properly credited.

\section{REFERENCES}

1. Khan U, Mason JM, Mecci M, Yiannakou Y. A prospective trial of temporary sacral nerve stimulation for constipation associated with neurological disease. Colorectal Dis 2014; 16(12): 1001-1009.

2. Atik B, Erkutlu I, Tercan $M$, Buyukhatipoglu $H$, Bekerecioglu $M$, Pence $S$. The effects of exogenous melatonin on peripheral nerve regeneration and collagen formation in rats. J Surg Res 2011; 166(2): 330-336.

3. Doth AH, Hansson PT, Jensen MP, Taylor RS. The burden of neuropathic pain: a systematic review and meta-analysis of health utilities. Pain 2010; 149(2): 338344.

4. Gaudet AD, Popovich PG, Ramer MS. Wallerian degeneration: gaining perspective on inflammatory events after peripheral nerve injury. $J$ Neuroinflammation 2011; 8(1)110.

5. Ellis $A$, Bennett $D L$. Neuroinflammation and the generation of neuropathic pain. Br J Anaesth 2013; 111(1): 26-37.

6. Bagdatoglu C, Saray A, Surucu HS, Ozturk H, Tamer L. Effect of trapidil in ischemia/reperfusion injury of peripheral nerves. Neurosurgery 2002; 51(1): 212-219; discussion 219-220.

7. Costigan M, Scholz J, Woolf CJ. Neuropathic pain: a maladaptive response of the nervous system to damage. Annu Rev Neurosci 2009; 32(1-32).

8. Dong $Y, X u Q$, Jia $X$, Li C, $X u D$, Jiao $Y$. Neuroprotective effect of thiamine triethylorthoformate conjugate against Parkinson disease in a mouse model. Trop J Pharm Res 2019; 18(5): 1041-1047

9. Negrao L, Almeida P, Alcino S, Duro H, Liborio T, Melo Silva U, Figueira R, Goncalves S, Neto Parra L. Effect of the combination of uridine nucleotides, folic acid and vitamin $B 12$ on the clinical expression of peripheral neuropathies. Pain Manag 2014; 4(3): 191-196.

10. den Hartigh LJ. Conjugated Linoleic Acid Effects on Cancer, Obesity, and Atherosclerosis: A Review of PreClinical and Human Trials with Current Perspectives. Nutrients 2019; 11(2).

11. Farghaly HS, Abd-Ellatief RB, Moftah MZ, Mostafa MG, Khedr EM, Kotb HI. The effects of dexmedetomidine alone and in combination with tramadol or amitriptyline in a neuropathic pain model. Pain Physician 2014; 17(2): 187-195.

12. Malmberg AB, Basbaum Al. Partial sciatic nerve injury in the mouse as a model of neuropathic pain: behavioral and neuroanatomical correlates. Pain 1998; 76(1-2): 215-222.

13. Colloca L, Ludman T, Bouhassira D, Baron R, Dickenson $A H$, Yarnitsky $D$, Freeman $R$, Truini A, Attal N, Finnerup $N B$ et al. Neuropathic pain. Nat Rev Dis Primers 2017; 3(17002).

14. Jaggi AS, Jain V, Singh N. Animal models of neuropathic pain. Fundam Clin Pharmacol 2011; 25(1): 1-28.

15. Scholz J, Woolf CJ. The neuropathic pain triad: neurons, immune cells and glia. Nat Neurosci 2007; 10(11): 1361 1368.

16. Park CK, Lu N, Xu ZZ, Liu T, Serhan CN, Ji RR. Resolving TRPV1- and TNF-alpha-mediated spinal cord synaptic plasticity and inflammatory pain with neuroprotectin D1. J Neurosci 2011; 31(42): 1507215085.

17. Tsujino H, Kondo E, Fukuoka T, Dai Y, Tokunaga A, Miki $K$, Yonenobu K, Ochi $T$, Noguchi K. Activating transcription factor 3 (ATF3) induction by axotomy in sensory and motoneurons: A novel neuronal marker of nerve injury. Mol Cell Neurosci 2000; 15(2): 170-182.

18. Obata K, Yamanaka H, Fukuoka T, Yi D, Tokunaga A, Hashimoto N, Yoshikawa H, Noguchi K. Contribution of injured and uninjured dorsal root ganglion neurons to pain behavior and the changes in gene expression following chronic constriction injury of the sciatic nerve in rats. Pain 2003; 101(1-2): 65-77.

19. Seijffers R, Mills $C D$, Woolf CJ. ATF3 increases the intrinsic growth state of DRG neurons to enhance peripheral nerve regeneration. J Neurosci 2007; 27(30): 7911-7920.

20. Schafers $M$, Sommer C. Anticytokine therapy in neuropathic pain management. Expert Rev Neurother 2007; 7(11): 1613-1627.

21. Zelenka M, Schafers $M$, Sommer C. Intraneural injection of interleukin-1beta and tumor necrosis factor-alpha into rat sciatic nerve at physiological doses induces signs of neuropathic pain. Pain 2005; 116(3): 257-263.

22. Leung L, Cahill CM. TNF-alpha and neuropathic pain--a review. J Neuroinflammation 2010; 7(27). 